\title{
Conclusion
}

Dany Baillargeon, professeur Université de Sherbrooke Dany.Baillargeon@USherbrooke.ca

Alexandre Coutant, professeur Université du Québec à Montréal coutant.alexandre@uqam.ca 
Une anecdote de terrain résume parfaitement le phénomène interrogé dans le cadre de ce numéro : depuis maintenant plus de quinze ans d'entretiens auprès de communicateur rices, l'expression de loin la plus récurrente est sans conteste « oh, mais tu sais, moi, j'ai un parcours/profil atypique ». Bien que depuis de nombreuses années, nous ayons assisté à des tentatives de standardisation dans les établissements d'enseignement et dans les associations professionnelles (Baillargeon et al., 2013 ; Lépine et David, 2014), souvent autant sous le coup d'une recherche de positionnement que d'un seul désir de rendre professionnelle la communication, les parcours professionnels des communicateur·rices demeurent hétérogènes. L'atypie serait-elle la typicité des métiers de la communication? Qu'est-ce que cet apparent paradoxe nous indique sur cette profession?

De nombreuses sources statistiques témoignent effectivement de la variété des postes et des provenances des praticien-nes des métiers de la communication. Ainsi, le classement des professions effectué lors du dernier recensement au Québec classe ces métiers au sein du groupe hétéroclite des « professions du domaine culturel et des communications », où se côtoient notamment relationnistes, acteurs et actrices, photographes et journalistes (Langlois, 2016). Malgré une évidente progression des formations spécialisées en communication (Jeffrey, Burton, 2012), les provenances des praticien'nes peuvent encore beaucoup varier, entre communication, gestion, sciences politiques, sociologie, psychologie, arts ou lettres (Coutant, 2009 ; David et Motulsky, 2010). L'atypie répondrait alors certainement à cette expertise de la « contorsion » (Dupouy, Fenot et Fukhura, 2015) qui semble, au premier abord, le seul élément réellement partagé par les communicateur rices.

Cette atypie s'illustre aussi dans la diversité des titres, appellations et noms de métiers plus ou moins pérennes, employés pour qualifier la fonction des communicateur-rices. La grande enquête sur les métiers de la communication au Québec dressait déjà ce constat (David et Motulsky, 2010) ${ }^{1}$, que le développement de nouveaux supports de communication n'a fait que renforcer (Coutant et Domenget, 2015).

En matière de trajectoire interne à la profession, il existe aussi une très forte mobilité entre les métiers, au cours d'une carrière ou entre la formation et la carrière (journalistes, relations publiques, publicité, etc.), le tout étant dû autant à des choix qu'à des contraintes face à certains secteurs bouchés ou à des défauts de reconnaissance des diplômes obtenus (Touati et Badulescu, 2014 ; Brizard-Kim, 2016). Les journalistes deviennent relationnistes ou producteur-rices de contenus (Baillargeon et al., 2016 ; Bernier et al., 2005) ; les mathmen deviennent les nouveaux et nouvelles communicateur rices à l'aune du big data (Couldry et Turow, 2014 ; Messinger, 2014) ; les responsables des RH endossent la fonction de communicateur-rice interne (Talal,

\footnotetext{
${ }^{1}$ Elle n'identifiait pas moins de 123 titres différents au sein des 679 répondant·es.
} 
2013). Soulignons aussi les fertilisations croisées entre politique, marketing et communication (Kugler, 2006 ; Stenger, 2012). Bref, autant d'indices que cette profession « échappe inévitablement à toute maitrise systématique » (Champy, 2009, p. 84).

Cette effervescence s'explique de nombreuses façons: relative jeunesse de la reconnaissance de la profession, difficulté à cerner son champ d'expertise propre, variété des fonctions impliquées, régulières transformations du secteur ${ }^{2}$ (Bernard, 2006 ; Lépine et al., 2014). La nature fondamentalement transdisciplinaire ou pluridisciplinaire de la communication appelle des professionnel-les aux compétences multiples et aux postures éthiques parfois contradictoires (Maas et al., 2017). Dans sa plus récente cartographie des champs de la discipline (Walter et al., 2018), la Conférence permanente des directeur rices des unités de recherche en sciences de l'information et de la communication circonscrivait dix champs avec autant de souschamps, tout en rappelant que « [dès] leur naissance et compte tenu de leurs origines (études cinématographiques, études littéraires, sciences de la documentation, sciences du langage, sémiologie, sociologie, etc.), les SIC se sont définies comme une... "interdiscipline" » (p. 12). Il en résulte que les praticien ·nes sont amené·es à compléter leur formation au long de leur carrière en intégrant des éléments hétéroclites. En somme, les professionnel·les de la communication offrent des temporalités biographiques (Bessin, 2009 ; Dubar, 2004) atypiques, marquées par des retours aux études, de la formation tout au long de la vie, des oscillations entre projets personnels et projets professionnels, emploi en organisation et travail autonome ou consultation. Les formations changent également régulièrement. La quête incessante d'une professionnalité aboutit en effet à une prolifération de formations et de certifications aux formats comme à la rigueur variables (de la Broise et Morillon, 2014). Les générations de diplômé es peuvent donc finir par ne plus se ressembler, voire se reconnaître. Ainsi voit-on des phénomènes de légitimation et de professionnalisation probants de cette atypie et davantage vecteur de difficultés : capital professionnel bâti par « hopping » (McLeod, O'Donohoe et Townley, 2011; Nixon, 2003 ; Pratt, 2006) ou par des dynamiques de liens marchands (Cochoy, 2012); forte rhétorique sur le savoir, à défaut d'une pratique standardisée (Alvesson, 2004) ; quête constante et ambivalente d'un idéal déontologique et éthique (Maas et al., 2017) ; polysémie et équivocité des titres d'emploi (David, Motulsky, 2010) ; identification (ou « désidentification ») à un statut professionnel (Jeffrey et Brunton, 2012) ; contestation du terme «professionnalisme » et croisement (nexus) d'importants questionnements théoriques et pratiques (Cheney et Lee Aschcraft, 2007).

\footnotetext{
${ }^{2}$ Qui concernent aussi bien les modes d'organisation du travail, les modèles théorético-pratiques guidant l'action, les outils de conception ou production, que les supports d'intervention.
} 
Ajoutons que les secteurs d'exercice viennent encore appuyer cette différenciation. Le ou la praticien'ne a souvent comme contrainte d'ajouter à ses compétences communicationnelles issues des formations initiales beaucoup de savoirs/savoirsfaire/savoirs-être spécifiques au secteur dans lequel il ou elle exerce : culture propre, forme organisationnelle en constant changement, spécificités techniques particulières, encadrement légal, supports usités ou prohibés, identités des parties prenantes...

Doit-on se réjouir de la porosité de ce marché du travail et laisser faire son homéostasie? Après tout, Menger (2018) ne cesse de démontrer le peu de valeur prédictive des formations pour anticiper la performance des futur es professionnel-les dans les métiers créatifs. L'atypie constituerait alors une adaptation logique aux spécificités de la tâche des communicateur-rices. Doit-on au contraire y voir la marque d'inadaptation des formations en communication, qui échapperaient à une partie au moins des compétences à transmettre pour les futur'es praticien·nes? Les enquêtes appuieraient davantage cette hypothèse.

Est-il possible de surmonter cette atypie ? La connaissant, doit-on intégrer dans les cursus des manières de préparer les futur'es praticien nes à apprendre à apprendre ?

C'est face à ces questions que le RESIPROC a décidé de solliciter des retours d'enquêtes empiriques : au-delà de ces constats statistiques, comment s'incarnent ces trajectoires? Comment sont-elles vécues par les acteur-rices, reconnues par leurs collaborateur-rices, etc. ? Plus particulièrement, mais de façon non exclusive, des réflexions portant sur les quatre axes suivants ont été sollicitées :

1. les différentes temporalités professionnelles des communicateur rices : temps de formation et temps professionnels; chevauchements de ces temporalités et dynamiques socioprofessionnelles les engendrant (mise à jour nécessaire des connaissances, mutation des pratiques sous le coup du numérique, mondialisation et mobilité, injonctions des associations professionnelles, etc.) ;

2. les hybridités des parcours : comment différentes professions se chevauchent, se contaminent, se combinent, avec les tensions que ces croisements génèrent et l'interrogation sur le fait que ces chevauchements participent ou non à la professionnalisation ;

3. l'(a)typie des parcours de formation, qu'ils soient institutionnalisés ou non, de même que les rationalités derrière leur existence ;

4. les vecteurs influant ces trajectoires, qu'ils soient d'ordres déontologiques, économiques, éthiques, politiques, sociotechniques.

Ces axes ont donné lieu à des propositions riches et diverses permettant d'observer cette nécessaire condition ou cette condition de nécessité que sont l'atypie et les hybridités des métiers de la communication. 


\section{Dans ce numéro}

Cette nécessaire atypie est particulièrement prégnante dans la proposition de Guillaume Le Saulnier, qui se concentre sur les policiers devenus communicants et des conditions de leur conversion professionnelle, et ce, dans un contexte d'intensification des activités de communications au sein des forces de l'ordre. Soutenu par une enquête ethnographique menée sur deux années au sein du Service d'information et de communication de la police (SICoP), Le Saulnier dépeint le parcours de ces officiers de communication, leurs quêtes de légitimité à travers l'acquisition de compétences - faites « sur le tas et sur le tard » (p. 33) avec seulement des formations généralistes - et des opérations de valorisation de leur statut marginal, qui leur fait «perdre leur qualité de policier aux yeux d'une partie de leurs pairs, mais aussi à leurs propres yeux» (p. 42). Ainsi, l'on découvre un environnement plus rébarbatif et contraignant que la vaste majorité des métiers de la communication, où le public imaginé des pairs est tout aussi présent que les publics auxquels doivent s'adresser ces communicatrices et communicateurs. Ainsi, Le Saulnier nous présente les atypies et hybridations d'une profession « au rebours d'une culture professionnelle qui postule une antinomie entre le rôle de policier et celui de communicant».

Pas très loin des préoccupations entourant la communication policière se dessine une autre territorialisation professionnelle, celle des communicateurs et communicatrices de l'Europe, que présente Sandrine Roginsky. Travaillant au sein ou en périphérie des institutions européennes, ceux-ci, à l'instar des officiers en communication de la SICoP, doivent de manière récurrente s'embarquer dans d'intenses rhétoriques de valorisation et de légitimité. Forte d'une soixantaine d'entretiens semidirigés et de l'analyse de profils Twitter et LinkedIn des personnels des institutions européennes chargés de fonctions de communication, d'agences d'affaires publiques ou d'agences de lobbying, cette recherche se penche sur les logiques de présentation de soi, d'exposition de soi et de justification de ces communicateurs et communicatrices de l'Europe. Après une cartographie des différentes instances accueillant ces personnels, Roginsky démontre que ceux-ci tentent de « se faire un nom », alors même que leur métier, à la frontière de plusieurs institutions, ne porte pas de nom. En résulte une pléthore de titres d'emploi souvent idiosyncrasiques, promouvant des compétences techniques (numériques) qui agissent comme passe-frontière : ces « dispositifs numériques ont certainement facilité le passage des communicateurs d'un monde à l'autre» (p. 55). Cette «difficile fabrique de l'éthos professionnel des communicateurs de l'Europe» (p. 66), est soumise à différentes tensions identitaires : éthos journalistiques vs posture du " geek »; le généraliste vs le spécialiste, sans compter que ce métier sert d'interface dans la conception et le suivi des politiques publiques, appelant une identification à la chose politique tout autant que communicationnelle. Roginsky conclut que la «[...] difficulté à faire émerger clairement un dénominateur commun à l'ensemble des professionnels qui font de la communication, tout ou partie, 
leur activité professionnelle est certainement à la fois cause et symptôme de la faible institutionnalisation de la fonction au sein de la bulle européenne ».

Dans un environnement professionnel volontiers présenté comme en mutation permanente, le regard rétrospectif proposé par Anaïs Théviot s'avère particulièrement judicieux. C'est au cas des professionnel-les de la communication politique numérique qu'elle s'intéresse, à la faveur des campagnes électorales pour la Présidence de la République Française menées de 2007 à 2017. Elle décrit la place que prennent progressivement ces métiers au sein du « tableau bien ordonné » (p. 78) que constituaient les campagnes. Au-delà des discours promotionnels accompagnant le numérique et ses promesses révolutionnaires, leur trajectoire laisse apparaître beaucoup de similarités avec les enjeux traditionnellement rencontrés par les métiers de la communication : même reconnaissance inaboutie, notamment visible dans l'absence de budgets importants dédiés ; même intégration progressive au sein de l'organisation existante, allant du ballotage au gré des forces en présence à la définition de services distincts, voire d'agences autonomes ; même porosité à l'arrivée de nouvelles compétences d'exécution (ici issues de l'informatique ou des engagements personnels dans les cultures numériques) masquant la relative stabilité des grands enjeux stratégiques auxquels les communicateurs et communicatrices sont censé es faire face; même focalisation des praticien-ne-s sur les compétences techniques ou l'expérience acquise sur le terrain. Ses constats amènent à penser le lien entre communication et hybridité à rebours : ce qui est observé relève davantage de praticien'nes spécialisé·es, ici en politique, qui intègrent des compétences communicationnelles extérieures à leurs cursus et trajectoires professionnelles habituel-les. L'enjeu est alors de faire prendre conscience que ce qu' ils voient comme des « enjeux communicationnels » complétant leur cœur de compétences peuvent se révéler plus centralement des «enjeux stratégiques » pour l'exercice de leur activité.

Vincent Brulois poursuit avec une réflexion sur l'engagement dans l'organisation, et ce, au regard du communicateur et de la communicatrice interne. Plus encore, il pose le postulat que pour engager et mobiliser les salarié·es d'une organisation par des activités de communication, engagement mesuré annuellement à coup d'enquêtes internes, les professionnel-les de la communication interne doivent également se sentir engagé'es. Eux-mêmes acteurs de cette organisation, comment conçoivent-ils leur propre engagement? Partant d'une réflexion sur les (nouvelles) attentes des salarié es - autonomie, réalisation de soi, accomplissement, sociabilité, projet de vie — qui œuvrent dans des organisations en constants changements et en tension, Brulois argue que «l'entreprise n'a de choix que de s'adresser à ses salariés afin de se saisir de leur potentiel de créativité et de leur capacité de mobilisation [...] pour "obtenir ce bon vouloir" et cet engagement nécessaire pour atteindre l'efficacité recherchée » (p. 13). En cela, le rôle de la communication interne est « donc de traduire les attentes des uns dans le vocabulaire des autres, de comprendre les métiers et les situations de travail, d'identifier et de partager les valeurs collectives, de maintenir le sens du travail 
en reliant les acteurs plus qu'en relayant l'information » (p. 17). Une telle description de tâches ne va pas sans un coûteux investissement personnel « ingrat », « usant » qui tient du «sacerdoce» pour maintenir ouverts les canaux de l'engagement. Grâce à cette plongée dans l'expérience des communicatrices et communicateurs internes, Brulois met en lumière l'hybridité de leurs rôles : passeur de sens et porteur de sens ; gestionnaire de l'image externe et des relations internes ; porte-voix du management et amplificateur de la parole des salariés. Dès lors, si revient aux communicateur rices internes le rôle de maintenir engagé, Brulois soutient que ceux-ci doivent faire un courageux "pari sur l'humain » et mettre en avant une «éthique de la discussion» difficile à soutenir dans ces organisations en surcharge informationnelle et en urgence permanente.

Arnaud Anciaux, Renaud Carbasse, Josianne Millette et Anne-Sophie Gobeil prennent à partie les mutations dans les trajectoires professionnelles en communication comme une traduction symptomatique des nouvelles exigences du capitalisme néolibéral. Les auteurs montrent que les promesses de liberté et d'autonomie entretenues dans les discours professionnels et managériaux ne sont pas sans conséquence et masquent des injonctions à la responsabilisation du travailleur quant à son succès, la flexibilité, la mobilité et la précarité accrues, doublées d'une instabilité des parcours et revenus. L'originalité de la proposition est qu'au lieu d'étudier une seule filière professionnelle, les auteurs préfèrent montrer les zones de convergence entre plusieurs métiers de la communication - journalisme et groupes médiatiques, relations publiques, gestionnaires de communauté —, donnant ainsi du poids à leur thèse d'un reflet des nouvelles valeurs du capitalisme dans les reconfigurations du travail en communication. Ils proposent ainsi de décloisonner l'étude des métiers de la communication pour mieux appréhender la transversalité des mutations, des hybridations et des atypies y prévalant. Ils proposent alors un programme de recherche en deux temps : «étudier ensemble les valeurs, les trajectoires professionnelles, les relations de travail et les conditions de pratiques communes aux différentes sphères de la communication» pour ensuite mieux «étudier les réactions différenciées des différentes sphères - et de ceux qui participent à leur construction - afin de comprendre les possibles résistances, violences perçues et revendications ».

Finalement, Élise Le Moing-Maas et Jean-Claude Domenget questionnent la construction professionnelle de la fonction de direction des communications (le ou la dircom). Ici encore, c'est par des atypies de parcours qu'émergent les dircoms, soulevant à nouveau des enjeux de légitimation d'une fonction hybride et instable. Les auteurs proposent quatre piliers pour comprendre ce travail de professionnalisation : l'éthos de la ou du dircom, particulièrement en regard des attentes de la direction générale ; la contribution de la fonction aux impératifs stratégiques de l'organisation ; les compétences certes techniques, mais davantage humaines, et assorties d'une «certaine impertinence»; des pratiques prudentielles, selon lesquelles et suivant Champy, les dircoms doivent mesurer l'impact de leurs décisions et celles du 
management, sans jamais les connaître à l'avance, en même temps qu' ils ·elles doivent s'engager dans ces décisions. En somme, «c'est justement en développant ces pratiques prudentielles que les dircoms pourront participer de la professionnalisation et de la reconnaissance de leur profession dans ses aspects politiques et sociaux (Dubar et al., 2015)» (p. 141).

En somme, on remarque, par la diversité des articles présentés dans ce numéro, que les atypies fondatrices des métiers de la communication, qui peuvent paraître anecdotiques, sont finalement constitutives de la profession. Plus encore, qu'ils ou elles soient officier de communication, communicatrice de l'Europe, communicateur numérique politique, communicatrice interne ou dircom, ces individus sont constamment en quête de légitimité, pour eux-mêmes autant que vis-à-vis de leurs pairs et leurs publics. Tentant de mobiliser des compétences plus techniques — souvent numériques - pour camper leur professionnalité, ces professionnel les de la communication évoluent dans un constant état d'incertitude identitaire, qu'ils et elles tentent d'adoucir en endossant la liberté imposée par ces métiers fragmentés. Ce qui revient à se demander ceci : alors même que toutes les organisations et institutions ont intensifié leurs activités de communication, comment peut-on comprendre cette constante quête de légitimité ? Est-ce l'incapacité à stabiliser la posture éthique fondamentale de la communicatrice ou du communicateur professionnel — qui sert-il vraiment et pourquoi le sert-il ? le peu d'assises théoriques et pragmatiques qui permettraient d'asseoir cette prééminence d'un savoir positif et cette utilité collective dont parlent les sociologies du travail ? Il est clair qu'avec la fragmentation des formes organisationnelles - organisation ouverte, impartition, co-working intraorganisationnel, etc. - la dilution des titres et pratiques professionnelles en communication ne fera que s'amplifier, rendant l'atypie encore plus typique.

\section{Bibliographie}

Alvesson, M. (2004). Knowledge work and knowledge-intensive firms. Oxford, Royaume-Uni : Oxford University Press.

Author, N. (2006). Marianne Kugler. Communication et organisation, 30, 278-281.

URL : http://journals.openedition.org/communicationorganisation/3498; DOI : 10.4000/communicationorganisation.3498

Baillargeon, D., Brulois, V., Coyette, C., David, M. D., Lambotte, F. et Lépine, V. (2013). Figures et dynamiques de la professionnalisation des communicateurs. Un miroir tendu aux associations en Belgique, en France et Canada. Communication \& Professionnalisation, 1, 12-32. 
Baillargeon, D., Coutant, A., Carignan, M.-E., Dionne, E. et Tourigny, M. (2017). Confrontations et convergences éthiques entre marketing et information autour de la publicité native. Communication \& Professionnalisation, 5, 28-53.

Bernard F. (2006). Les SIC, une discipline de l'ouverture et du décloisonnement. In Arlette Bouzon (dir.), La Communication organisationnelle en débat. Champs, concepts, perspectives. Paris, France : L'Harmattan, 33-46.

Bernier, M.-F., Demers, F., Lavigne, A., Moumouni, C. et Watine, T. (2005). Pratiques novatrices en communication publique. Journalisme, relations publiques et publicité. Québec, Canada : Presses de l'Université Laval.

Bessin, M. (2009). Parcours de vie et temporalités biographiques : quelques éléments de problématique. Informations sociales, 6(156), 12-21.

Brizard-Kim K. (2016). Quand les professionnels indépendants réinventent l'agence de communication Collectifs de freelances, communautés de coworkers, entreprises coopératives. Communication \& Professionnalisation, 4, 143-161.

Broise (de la), P. et Morillon, L. (2014). Des pratiques aux formations professionnelles, les liaisons dangereuses du marketing et de la communication. In V. Lépine, C. Millet-Fournier et M. Martin-Juchat (Éds.), Acteurs de la communication des entreprises et organisations : pratiques et perspectives, Grenoble, France : Presses universitaires de Grenoble, 165-180.

Brulois, V., Carignan, M.-E., David M. D. et Errecart, A. (2016). Dynamiques de la professionnalisation en communication : entre ruptures et continuités, prescription et émancipation. Communication \& Professionnalisation, 4.

Champy, F. (2009). La sociologie des professions. Paris, France: Presses universitaires de France.

Cheney, G. et Lee Ashcraft, K. (2007). Considering "The Professional" in Communication Studies: Implications for Theory and Research Within and Beyond the Boundaries of Organizational Communication. Communication Theory, 17(2), 146-175.

Cochoy, F. (2012). Du lien marchand, comment le marché fait société : essai(s) de sociologie économique relationniste. Toulouse, France : Presses universitaires du Mirail.

Couldry, N. et Turow, J. (2014). Advertising, Big Data and the Clearance of the Public Realm: Marketers' New Approaches to the Content Subsidy. International Journal of Communication, 8(17). URL : http://ijoc.org/index.php/ijoc/article/ view/2166 
Coutant A. (2009). Les cordonniers sont toujours les plus mal chaussés : les difficultés d'accord entre les professionnels de la communication. Communication \& Organisation, 35, 227-239.

Coutant A. et Domenget J.-C. (2015). Le communicateur bousculé par le numérique. Quelles compétences à transmettre ? Communication \& Professionnalisation, 3.

David M. D. et Motulsky B. (2010). La grande enquête : vers une meilleure connaissance des pratiques en communication dans les organisations canadiennes. URL : http://www.crp.uqam.ca/pages/docs/centres/Resultats_GE_2011.pdf

Dubar, C. (2004). Régimes de temporalités et mutation des temps sociaux. Temporalités, 1, 118-129.

Dubar, C., Tripier, P. et Broussard, V. (2015). Sociologie des professions. Paris, France : Armand Colin.

Dupouy, S., Fenot, P. et Fukuhara, K. (2015). Communicants : une théorie de la contorsion? Sociologies pratiques, 30.

Jeffrey, L. et Burton, M. (2012). Professional identity: how communication management practitioners identify with their industry. Public Relations Review, 38, 156158.

Langlois, S. (2016). Le Québec change. Chroniques sociologiques. Montréal, Québec : Del Busso Éditeur.

Lépine, V. et David, M. D. (2014). Pratiques et réflexions autour des dispositifs d'apprentissage et de formation des communicateurs. Communication \& Professionnalisation, 2.

Lépine, V., Martin-Juchat, F. et Millet-Fourrier, C. (2014). Acteurs de la communication des entreprises et organisations : pratiques et perspectives. Grenoble, France : Presses universitaires de Grenoble.

Maas, E., Domenget, J.-C. et Catellani, A. (2017). Professionnalisation et éthique de la communication. Des principes à la formation. Communication \& Professionnalisation, 5.

McLeod, C., O’Donohoe, S. et Townley, B. (2011). Pot Noodles, Placements and Peer Regard: Creative Career Trajectories and Communities of Practice in the British Advertising Industry. British Journal of Management, 22(1), 114-131.

Menger, P.-M. (2018). Le talent en débat. Paris, France : PUF.

Messinger, B. (2014). From Mad Men to Math Men. URL : https://www.ama.org/ publications/MarketingInsights/Pages/from-mad-men-to-math-men.aspx 
Nixon, S. (2003). Advertising Cultures: Gender, Commerce, Creativity. London, Royaume-Uni : Sage publications.

Pratt, A. C. (2006). Advertising and Creativity, a Governance Approach: A Case Study of Creative Agencies in London. Environ Plan A, 38(10), 1883-1899.

Stenger, T. (dir.). (2012). Le marketing politique. Paris, France : CNRS éditions.

Talal, M. (2013). Add Value to Internal Communication through Human Resources Management. Valahian Journal of Economic Studies, 4(4), 21-30.

Touati, Z. et Badulescu, C. (2014). Employabilité, insertion professionnelle et mobilité des jeunes diplômés dans les métiers de la communication en France. Communication \& Professionnalisation, 2, 102-122.

Walter, J., Douyère, D., Bouillon, J.-L. et Ollivier-Yaniv, C. (2018). Dynamiques des recherches en sciences de l'information et de la communication. URL: http://cpdirsic.fr/wp-content/uploads/2018/09/dynamiques-des-recherches-sicweb-180919.pdf 